\title{
Concerning the Mechanism of Reflection of Electromag- netic Waves From an Inhomogeneous Lossy Plasma
}

\author{
James R. Wait \\ Central Radio Propagation Laboratory \\ National Bureau of Standards, Boulder, Colo.
}

(Received January 21, 1965)

\begin{abstract}
Reflection of electromagnetic waves from a stratified lossy inhomogeneous plasma is discussed. The profile for the conductivity is idealized as an exponential curve with a superimposed Gaussianshaped perturbation. By examining the change in the calculated reflection coefficient for various locations of the perturbation, some insight into the reflection process is gained. In particular, it is shown that reflection does not take place at a single level but, instead, a wide range of levels is important. The parameters of the problem are chosen to be representative of the $D$ layer of the ionosphere and the wavelengths cover the range $10 \mathrm{~km}$ to $30 \mathrm{~km}$.
\end{abstract}

\section{Introduction}

It is well known that the reflection coefficient of a stratified medium is determined explicitly by the profile of the complex dielectric constant. For example, if the profiles of electron density and collision frequency in the lower ionosphere are specified, it is possible to calculate the reflection coefficient for an obliquely incident electromagnetic wave. Although there is now a vast literature devoted to this general subject, some questions concerning the nature of the reflection process remain unanswered. For example, the determination of the effective reflection level in the ionosphere for VLF radio waves is not a clear-cut task. In fact, even the concept that waves are reflected at some specific level is open to question.

It is the purpose of the present paper to investigate which levels in the lower ionosphere are most influential in determining the reflection coefficient. A number of simplifications are made in order that extraneous considerations are removed. For example, in the lowest ionosphere in the daytime it is assumed that the following inequalities hold:

(a) Electron collision frequency $\nu_{e} \gg$ electron gyrofrequency $\Omega_{e}$.

(b) Ion collision frequency $\nu_{i} \gg$ angular wave frequency $\omega$.

(c) Density of neutral particles $N_{0} \gg$ both density of electrons $N_{e}$ and density of ions $N_{i}$.

Under these conditions, it is then a straightforward matter [Wait, 1962] to show that the ionized medium may be characterized by a scalar complex dielectric constant $\epsilon(z)$ which is a function of height $z$ of the form

$$
\frac{\epsilon(z)}{\epsilon_{0}}=1-\frac{i}{L(z)},
$$

where

$$
\frac{1}{L(z)}=\frac{e^{2}}{\epsilon_{0} \omega}\left[\frac{N_{e}}{m_{e} \nu_{e}}+\frac{N_{i}}{m_{i} \nu_{i}}\right]
$$

In the above,

$\epsilon_{0}$ is the dielectric constant of free space,

$e$ is the charge of the electron and the ion, and

$m_{e}$ and $m_{i}$ are the masses of the electrons and the ions, respectively.

The only difference between this formulation and a previous one [Wait and Walters, 1963], is that here the ions are considered explicitly. The form of the dielectric constant $\epsilon(z)$ is just the same. Thus, the numerical method for calculating the reflection coefficient is identical and need not be described here.

\section{The Idealized Profile}

To illustrate the salient features, it is assumed that the conductivity parameter $1 / L(z)$ of the $D$-layer may be written in the following analytical form:

$$
\frac{1}{L(z)}=\frac{1}{L_{o}}\left[\exp (\beta z)+A_{0} \exp \left[-\left(\frac{z-F}{D}\right)^{2}\right]\right] \text {. }
$$




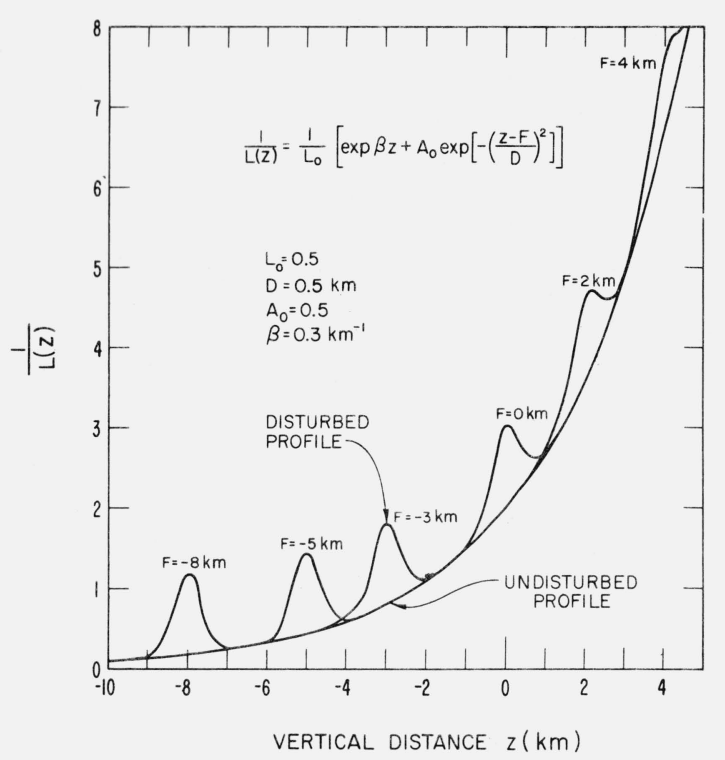

FIGURE 1. The idealized profile of the inhomogeneous lossy plasma. The ordinate is proportional to the conductivity of the medium as a function of height above and below the reference level at $z=0$. The perturbation is shown at a number of levels from $z=F=-8 \mathrm{~km}$ to $+4 \mathrm{~km}$.

The resulting exponential profile obtained by setting $A_{0}=0$ may be described as the "undisturbed profile." Typically, for daytime conditions, $\beta$ would be about $0.3 \mathrm{~km}^{-1}$ when $z$ is measured in $\mathrm{km}$ above some reference level at $z=0$. The second term in square brackets is then regarded as a perturbation which is centered at $z=F$, while its vertical extent is determined by the parameter $D$. In an abnormally ionized $D$ region, such as discussed by Crain and Booker [1964], the ions can contribute significantly to the perturbation. The function $1 / L(z)$, as defined above, is plotted in figure 1 as a function of height $z$ where $L_{0}$ is set equal to $1 / 2$. The smooth curve in this figure corresponds to the undisturbed exponential profile. The effect of locating the perturbation at various heights is illustrated in figure 1 by choosing a sequence of $F$ values from $-8 \mathrm{~km}$ to $+4 \mathrm{~km}$, while $D$ is chosen to be $0.5 \mathrm{~km}$ and $A_{0}$ is taken to be 0.5 .

It is stressed that the perturbation, as described above, is highly idealized. It is not intended that it should represent the $D$-layer under actual disturbed conditions.

\section{Description of Numerical Data}

It is now contended that considerable insight into the reflection process is obtained by examining the changes in the reflection coefficient as the perturbation is moved along the profile as indicated in figure 1 . Results of this kind are shown in figures $2 a$ and $2 b$ for the amplitude and phase of the reflection $R$ for a vertically polarized incident wave. The cosine $C$ of the angle of incidence is denoted on the curves.
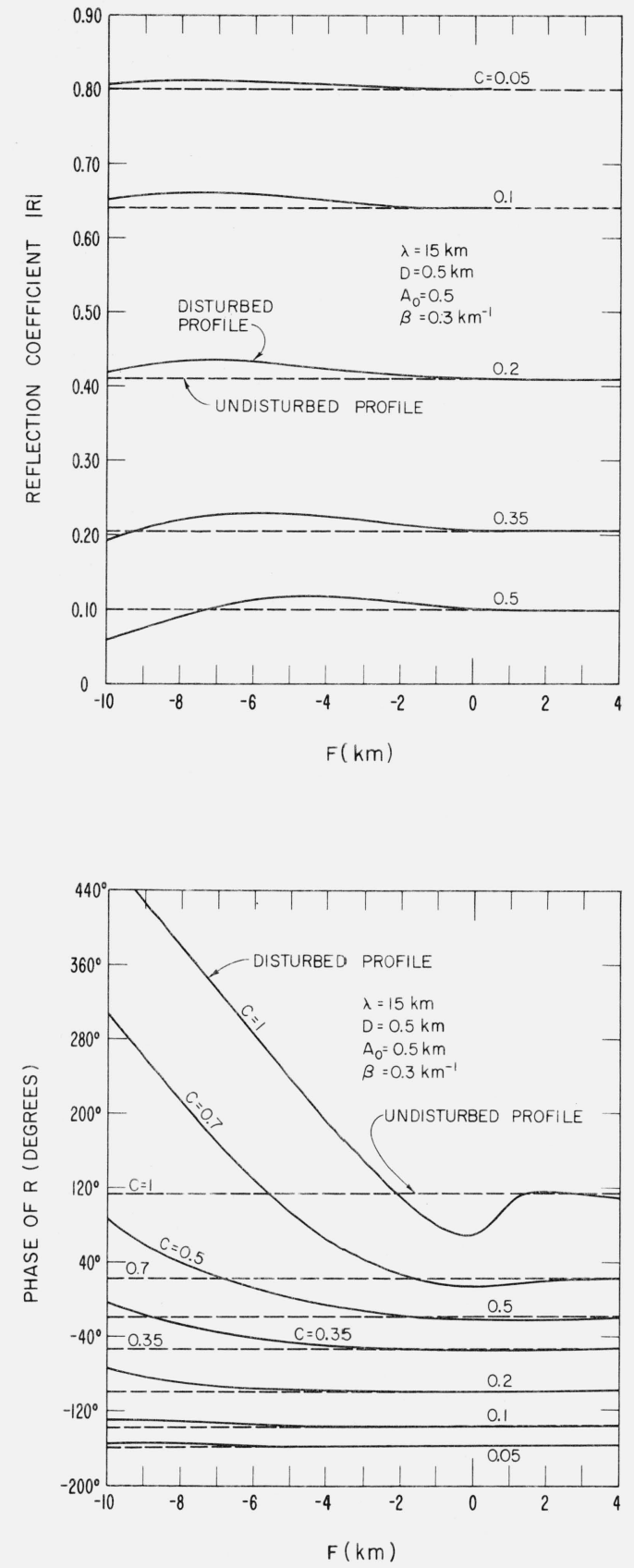

FIGURES $2 \mathrm{a}$ and $2 \mathrm{~b}$. The (vertically polarized) reflection coeffcient as a function of $\mathrm{F}$ which is the vertical level of the perturbation.

Also, as indicated, the wavelength $\lambda$ is taken to be $15 \mathrm{~km}$ corresponding to a frequency of $20 \mathrm{kc} / \mathrm{s}$. The results for the undisturbed exposential profile are shown in figures $2 \mathrm{a}$ and $2 \mathrm{~b}$ by dashed lines. Following previous conventions [Wait and Walters, 1963], the phase is referred to the level $z=0$.

It is evident from the curves in figure $2 a$ that the perturbation only has an influence on $|R|$ if it is located below the level $z=F=0$. For $F$ in the interval from 
about -1 to -6 , the reflection coefficient is increased somewhat. Thus, the perturbation at these levels actually enhances the reflection process. Of course, for increasingly larger negative values of $F$, the opposite tendency becomes evident. Then the perturbation acts as an absorber, tending to diminish the reflection coefficient.

It is suggested that the "levels of reflection" in the unperturbed profile are characterized by the interval in $F$ where $|R|$ for the perturbed profile exceeds $|R|$ for the unperturbed profile by some detectable increment. For example, when $C=0.2$, the "reflection levels" extend from about $F=-2$ to $-10 \mathrm{~km}$. In this range, $|R|$ for the perturbed profile exceeds $|R|$ for the undisturbed profile by at least 5 percent. It is interesting to compare this deduction with a statement of Crain and Booker [1964] that the reflection takes place in the height range near the level given by

$$
\frac{e^{2}}{\epsilon_{0} \omega}\left(\frac{N_{e}}{m_{e} \nu_{e}}+\frac{N_{i}}{m_{i} \nu_{i}}\right)=2 C^{2} .
$$

For the undisturbed exponential profile, this is equivalent to solving the equation

$$
2 \exp (\beta F)=2 C^{2} .
$$

Thus, taking $\beta=0.3$ and $C=0.2$ as in the above example, it is readily found that

$$
F=-11 \mathrm{~km} \text {. }
$$

Crain and Booker [1964] do not explain how they arrive at their criterion for the reflection level but, presumably, it is related to the phase integral approach of Eckersley [1932]. The latter is straightforward for lossless media at high frequency where such geometrical-optical notions are meaningful. For lower frequencies in relatively lossy media, the phase integral method loses its validity. This would appear to be the reason for the apparent discrepancy between the Crain and Booker "reflection level" at $F=-11 \mathrm{~km}$ and the results in figure $2 a$ which would indicate that reflection takes place at a range of levels from about $-2 \mathrm{~km}$ to $-10 \mathrm{~km}$. The difference between the phase integral approach and the full wave solution becomes even greater for angles nearer grazing incidence. In general, the reflection level predicted by the Crain and Booker criterion is at the bottom or somewhat below the range of levels in the lower ionosphere where VLF radio waves are reflected. However, this view does not modify the conclusions regarding the importance of ions which was the principal topic in the paper by Crain and Booker [1964]. Furthermore, their "reflection level" is probably quite representative at the height region where the bulk of the energy is actually returned.

\section{Connection With Mode Theory}

An important parameter occurring in the theory of waveguide propagation of VLF radio waves is the complex quantity $\alpha$ [e.g., Wait and Spies, 1964]. In terms of the reflection coefficient $R$, it is defined by

$$
R=-\exp (\alpha C),
$$

where $C$ is the cosine of the angle of incidence. Writing $\alpha=\alpha_{1}+i \alpha_{2}$, the real quantities $\alpha_{1}$ and $\alpha_{2}$ are shown in figures $3 \mathrm{a}$ and $3 \mathrm{~b}$ for $C=0.1$ and 0.2 , where we have used the perturbed exponential model described above. The magnitude of $\alpha_{1}$ is approximately proportional to the attenuation of the dominant mode in the earth-ionosphere waveguide. Thus, for $F$ greater than zero, the attenuation is not influenced by the perturbation. However, for a range of $F$ from about -1 to -10 or so, there is a detectable change in the attenuation rate.

The variation of the reflection parameters $\alpha_{1}$ and $\alpha_{2}$ as a function of the angle of incidence is shown in figures $4 \mathrm{a}$ and $4 \mathrm{~b}$ for a range of $F$ values. It is important to observe that the functional dependence on $C$ is markedly changed when the perturbation is lowered from $F=0$ to $F=-10 \mathrm{~km}$. The consequence is that the relative attenuation of the first and second order waveguide modes is appreciably modified by a low-lying perturbation of a smooth undisturbed profile. The general tendency is to increase the relative attenuation of the second order mode as the perturbation is lowered. The phase parameter $\alpha_{2}$ in figure $4 \mathrm{~b}$ indicates that the perturbation generally increases the phase velocity of the dominant waveguide modes.
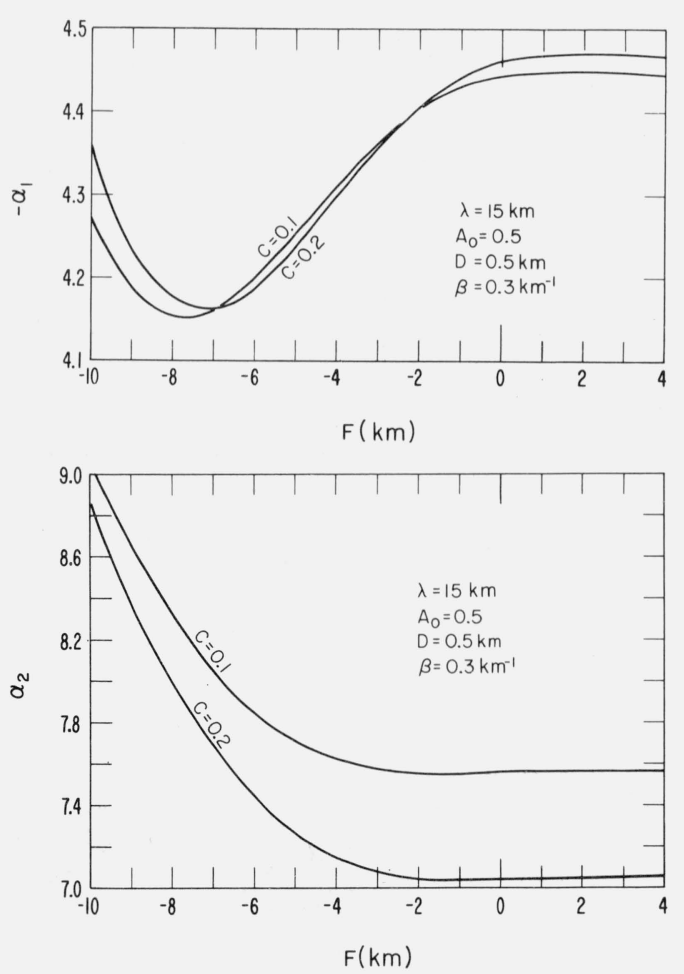

Figures $3 \mathrm{a}$ and $3 \mathrm{~b}$. The reflection parameters $\alpha_{1}$ and $\alpha_{2}$ which are defined by $R=-\exp (\alpha C)$ where $\alpha=\alpha_{1}+\mathrm{i} \alpha_{2}$. 

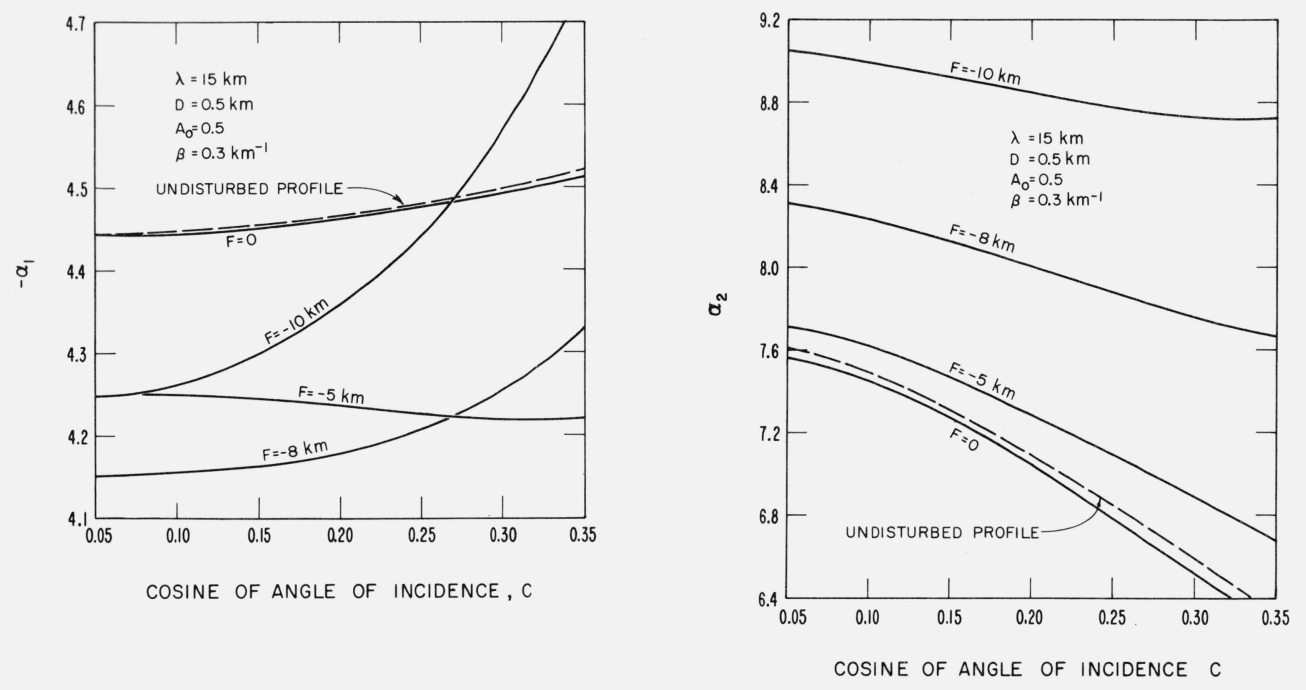

Figures $4 \mathrm{a}$ and $4 \mathrm{~b}$. The reflection parameters as a function of the cosine of the angle of incidence.

For example, at a frequency of $20 \mathrm{kc} / \mathrm{s}$, the phase velocity (relative to $c$ ) of mode $n=1$ changes from $\underline{0.9986}$ to $\underline{0.9989}$ as the location of the perturbation moves from $F=0$ to $-10 \mathrm{~km}$. In this case, it is assumed that the $C=0.15$ and the reference level $(z=F=0)$ in the ionosphere is located at a height $h=70 \mathrm{~km}$ above a perfectly conducting earth. Other quantitative estimates of this kind may be obtained by using the waveguide parameter curves issued in a recent NBS publication [Wait and Spies, 1964].

\section{Wavelength Dependence}

The results discussed above all pertain to a wave-

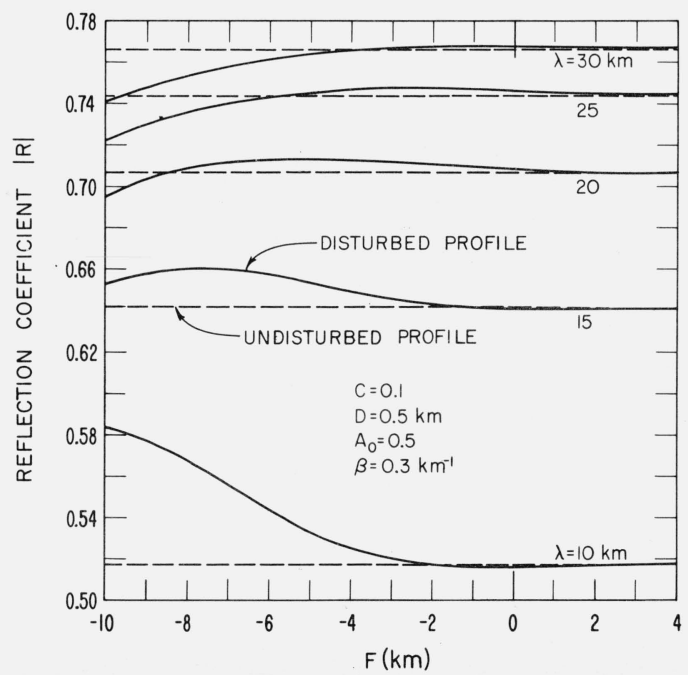

length of $15 \mathrm{~km}$. To illustrate the wavelength dependence, the amplitude and phase of the reflection coefficient are plotted as a function of $C$ in figures $5 \mathrm{a}$ and $5 \mathrm{~b}$, respectively, for wavelengths extending from $\lambda=10$ $\mathrm{km}$ to $30 \mathrm{~km}$. The function $L_{0}$ is defined by $L_{0}=30 / \lambda$ where $\lambda$ is the wavelength in $\mathrm{km}$. It is interesting to observe that the curve in figure $5 \mathrm{a}$ for $\lambda=10 \mathrm{~km}$ has reflection levels extending from $F=-2 \mathrm{~km}$ to well below $F=-10 \mathrm{~km}$. The Crain-Booker [1964] reflection level would be found by solving

$$
3 \exp (\beta F)=2 C^{2}=0.02,
$$

which yields $F=-16.5 \mathrm{~km}$ which is not far below the physically important height range where the reflection actually takes place.

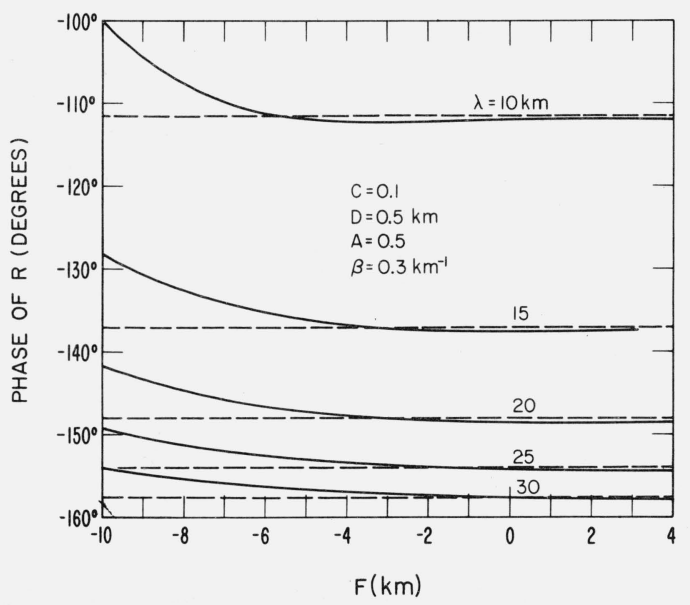

FIGURES $5 \mathrm{a}$ and $5 \mathrm{~b}$. The wavelength dependence of the reflection coefficient. 


\section{Conclusions}

The graphical results given in this paper would indicate that reflection does not take place at a discrete level in an inhomogeneous lossy medium with a smooth profile of its dielectric constant. In fact, the wave is reflected at a range of levels in the medium. The upper limit is determined where the incident wave has been sufficiently attenuated by a result of conduction losses. This is in contrast to the mechanism which produces reflection in a lossless inhomogeneous medium. In that case, there is a fairly well determined reflection level which is located where the vertical propagation constant vanishes.

It is important that the reflection process be understood if experimental data on VLF fields are to be interpreted in relation to the properties of the lower ionosphere. All too often, variations of the amplitude and the phase of the V.LF field are ascribed to changes in the parameters of some simplified model which bear little resemblance to the actual physical changes which have taken place.

The author thanks Mrs. L. C. Walters who programmed the digital computer for the calculations discussed in this paper. The work was supported by the Advanced Research Projects Agency under ARPA Order No. 183-62.

\section{References}

Crain, C. M., and H. G. Booker (Nov. 1, 1964), The effects of ions on low frequency propagation in an abnormally ionized atmosphere, J. Geophys. Res. 69, No. 21, 4713-4716.

Eckersley, T. L. (1932), Radio transmission problems treated by phase integral methods, Proc. Roy. Soc. A136, 499-527.

Wait, J. R. (1962), Electromagnetic waves in stratified media (Macmillan Co., New York).

Wait, J. R., and K. P. Spies (Dec. 1964), Characteristics of the earth-ionosphere waveguide for VLF radio waves, NBS Tech. Note No. 300.

Wait, J. R., and L. C. Walters (1963), Reflection of VLF radio waves from an inhomogeneous ionosphere, J. Res. NBS 67D (Radio Prop.), Part I. Exponentially varying isotropic model, No. 3, 361-367 (May-June); Part II. Perturbed exponential model, No. 5, 519-523 (Sept.-Oct.); Part III. Exponential model with hyperbolic transition, No. 6, 747-752 (Nov.-Dec.).

\section{Ådditional Reference}

Johler, J. R., and L. A. Berry (1965), On the effect of heavy ions on LF propagation (to be issued as an NBS Technical Note). 